\title{
On the Morse-Sard property and level sets of Sobolev and BV functions
}

\author{
Jean Bourgain, Mikhail V. Korobkov and Jan Kristensen
}

\begin{abstract}
We establish Luzin $N$ and Morse-Sard properties for $\mathrm{BV}_{2}$ functions defined on open domains in the plane. Using these results we prove that almost all level sets are finite disjoint unions of Lipschitz arcs whose tangent vectors are of bounded variation. In the case of $\mathrm{W}^{2,1}$ functions we strengthen the conclusion and show that almost all level sets are finite disjoint unions of $\mathrm{C}^{1}$ arcs whose tangent vectors are absolutely continuous along these arcs.
\end{abstract}

\section{Introduction}

For $\mathrm{C}^{2}$-smooth functions $v: \Omega \rightarrow \mathbb{R}$ that map an open subset $\Omega$ of $\mathbb{R}^{2}$ into $\mathbb{R}$, the classical Morse-Sard theorem [30], [37] (see also Brown [8] for a precursor and [16] for a more general exposition) guarantees that the set of critical values is negligible in the sense that

$$
\mathcal{L}^{1}\left(v\left(Z_{v}\right)\right)=0,
$$

where $\mathcal{L}^{1}$ is the one-dimensional Lebesgue measure on $\mathbb{R}$ and $Z_{v}$ is the critical set of $v$ defined as $Z_{v}=\{x \in \Omega: \nabla v(x)=0\}$. Whitney demonstrated [38] that the $\mathrm{C}^{2}$-smoothness condition in the above assertion cannot be dropped. Namely, he constructed a $\mathrm{C}^{1}$-smooth function $v:(0,1)^{2} \rightarrow \mathbb{R}$ such that the set $Z_{v}$ of critical points contains an arc on which $v$ is not constant (subsequently called a Whitney arc). However, some analogs of the Morse-Sard theorem remain valid for functions lacking the required smoothness in the classical theorem. Although (1.1) may be no longer valid then, Dubovitskiu [14] obtained some results on the structure of the level sets in the case of reduced smoothness (also see [5]).

Another direction of research was the generalization of the Morse-Sard theorem to functions in Hölder and Sobolev spaces (for example, see [4], [5], [12], [17], and [31]). In particular, we mention the work [12] of De Pascale (see also [17])

Mathematics Subject Classification (2010): Primary 58K05; Secondary 46E35.

Keywords: $\mathrm{BV}_{2}$ and $\mathrm{W}^{2,1}$ functions, Luzin $N$ property, Morse-Sard property, level sets. 
where it was shown that (1.1) holds when $v \in \mathrm{W}_{\text {loc }}^{2, p}(\Omega)$ for $p>2$. Note that in this case $v$ is $\mathrm{C}^{1}$-smooth by virtue of the Sobolev imbedding theorem, and so the critical set is defined as usual.

We should mention that all the above mentioned papers in fact concern the general multidimensional case and that we, for expository purposes and in line with the results presented in this paper, only commented on the particular case of real-valued functions defined on a plane domain. However, there are also some results that only concern, or at least have so far only been established for this particular case. For example, the following Morse-Sard-type theorem was obtained by Pogorelov (see Chapter 9, Section 4 in [34]): For a $\mathrm{C}^{1}$ function $v: \Omega \subset \mathbb{R}^{2} \rightarrow \mathbb{R}$ defined on an open planar domain $\Omega$, the equality (1.1) holds if for any linear function $\ell: \mathbb{R}^{2} \rightarrow \mathbb{R}$ the sum $v+\ell$ satisfies the maximum principle (see also [19] for another proof of this result). In particular, the equality (1.1) holds if the gradient range $\nabla v(\Omega)$ has no interior points (see [20] and [22] for a study of such functions in the planar case and [21] for the multidimensional case).

In the paper [9] it was proved that for functions $v \in \mathrm{W}_{\text {loc }}^{2, p}\left(\mathbb{R}^{2}\right)$ with $p>1$ there are no Whitney arcs (see also [18] on the same subject in the context of Hölder spaces, and [10] and [11] for further references and results on Whitney arcs).

Landis [27] proved that the equality (1.1) holds if $v: \Omega \subset \mathbb{R}^{2} \rightarrow \mathbb{R}$ is a difference of two convex functions (sometimes called a d.c. function), a result which answered a question raised previously by Pogorelov. Pavlica and Zajíček [32] presented a detailed and modern proof of the result of Landis. Moreover, they proved in [32] that the equality (1.1) holds more generally for Lipschitz functions of class $\mathrm{BV}_{2, \mathrm{loc}}(\Omega)$, where $\operatorname{BV}_{2, \text { loc }}(\Omega)$ is the space of functions $v \in \mathrm{W}_{\text {loc }}^{1,1}(\Omega)$ for which all partial (distributional) derivatives of the second order are signed Radon measures on $\Omega$.

In this paper we extend the last result to the case of any $\mathrm{BV}_{2}$ function defined on a planar domain (without the additional Lipschitz assumption, see Theorem 4.1). Since such functions need not be everywhere differentiable one must pay special attention to the definition of the critical set: it is known by work of Dorronsoro [13] (see Lemma 4.2 below for a precise statement), that in general a function $v \in$ $\mathrm{BV}_{2, \text { loc }}(\Omega)$ admits a continuous representative which is differentiable outside a 1 -rectifiable set, and that has "half-space differentials" $\mathcal{H}^{1}$-almost everywhere. We include in the critical set $Z_{v}$ the points $x \in \Omega$ such that one of the "half-space differentials" is zero at $x$. As a consequence our critical set can be strictly larger than the one defined in [32]. The precise definition of $Z_{v}$ is given at the beginning of Section 3.

Our main result, contained in Theorem 3.1 and Corollary 3.2, is to establish the Luzin $N$ property with respect to $\mathcal{H}^{1}$ for $\mathrm{BV}_{2}$ functions on planar domains. More precisely, we show that if $v$ is $\mathrm{BV}_{2}$ on an open domain $\Omega \subset \mathbb{R}^{2}$ with Lipschitz boundary, then for any $\varepsilon>0$ there exists $\delta>0$ such that for all subsets $E \subset \Omega$ with 1-dimensional Hausdorff content $\mathcal{H}_{\infty}^{1}(E)<\delta$ we have $\mathcal{L}^{1}(v(E))<\varepsilon$. In particular, it follows that $\mathcal{L}^{1}(v(E))=0$ whenever $\mathcal{H}^{1}(E)=0$. So the image of the exceptional "bad" set, where neither the differential nor the half-space differentials are defined, has zero Lebesgue measure. This ties in nicely with our definition of the critical set and our version of the Morse-Sard result for $\mathrm{BV}_{2}$ functions on the plane. 
Finally, using these results we prove that almost all level sets of $\mathrm{BV}_{2}$ functions defined on open domains in the plane, are finite disjoint unions of Lipschitz arcs whose tangent vectors have bounded variations (Theorem 6.1 and Corollary 6.2). In the $\mathrm{W}^{2,1}$ case we can strengthen the conclusions and show that almost all level sets are finite disjoint unions of $\mathrm{C}^{1}$ arcs whose tangent vectors are absolutely continuous functions (Theorem 5.1 and Corollary 5.2).

The results presented here have recently found some applications in fluid mechanics (see [23]-[25]).

After this work was completed we learned that [1] has also recently established the Morse-Sard property for $\mathrm{W}^{2,1}$ functions on the plane.

When this paper was ready for publication, we obtained an $n$-dimensional version of most of these results for $v \in \mathrm{BV}_{n}\left(\mathbb{R}^{n}\right)$, see [7].

Finally we wish to thank the referee for many useful comments that helped us to improve the presentation.

\section{Preliminaries}

Throughout this paper $\Omega$ denotes an open subset of $\mathbb{R}^{2}$. By a domain we mean an open connected set. For a general subset $E \subset \mathbb{R}^{2}$, we let $\mathrm{Cl} E$ stand for its closure, Int $E$ for its interior, and $\partial E$ for its boundary.

For a distribution $T$ on $\Omega$ denote by $D_{i} T, i=1,2$, the distributional partial derivatives of $T$, and write $D T=\left(D_{1} T, D_{2} T\right)$. For signed or vector-valued Radon measures $\mu$ we denote by $\|\mu\|$ the total variation measure of $\mu$ (in fact, we shall encounter measures valued in $\mathbb{R}^{2}$ and in $\mathbb{R}^{2 \times 2}$ and in both cases we use the standard Euclidean norms). The space $\mathrm{BV}(\Omega)$ is as usual defined as consisting of those functions $f \in \mathrm{L}^{1}(\Omega)$ whose distributional partial derivatives $D_{i} f$ are Radon measures with $\left\|D_{i} f\right\|(\Omega)<\infty$ (for detailed definitions see [15]). As a consequence of the Radon-Nikodym theorem we have for any $f \in \mathrm{BV}(\Omega)$ the polar decomposition of the distributional derivative $D f(E)=\int_{E} \nu \mathrm{d}\|D f\|$, where $\nu: \Omega \rightarrow \mathbb{S}^{1}$ is a Borel vector field valued in the unit sphere $\mathbb{S}^{1} \subset \mathbb{R}^{2}$, and $\|D f\|$ is the total variation measure of $D f$. The Radon-Nikodym derivative of $D f$ with respect to the Lebesgue measure $\mathcal{L}^{2}$ is denoted by $\nabla f$. The norm is $\|f\|_{\mathrm{BV}(\Omega)}=\|f\|_{\mathrm{L}^{1}(\Omega)}+\|D f\|(\Omega)$, and we write $\|v\|_{\mathrm{BV}}$ instead of $\|v\|_{\mathrm{BV}\left(\mathbb{R}^{2}\right)}$.

Our main results concern functions belonging to the space $\operatorname{BV}_{2}(\Omega)$ defined as those functions $v \in \mathrm{L}^{1}(\Omega)$ such that $D_{1} v, D_{2} v \in \mathrm{BV}(\Omega)$. We use the norm

$$
\|v\|_{\mathrm{BV}_{2}(\Omega)}=\|v\|_{\mathrm{L}^{1}(\Omega)}+\|\nabla v\|_{\mathrm{L}^{1}(\Omega)}+\left\|D^{2} v\right\|(\Omega)
$$

on $\mathrm{BV}_{2}(\Omega)$. Also functions in the Sobolev spaces $\mathrm{W}^{1,1}(\Omega)=\left\{f \in \mathrm{L}^{1}(\Omega): D_{i} f \in\right.$ $\left.\mathrm{L}^{1}(\Omega), i=1,2\right\}, \quad \mathrm{W}^{2,1}(\Omega)=\left\{v \in \mathrm{L}^{1}(\Omega): D_{i} f \in \mathrm{W}^{1,1}(\Omega), i=1,2\right\}$ play prominent roles in our results.

It is known that each function $v \in \mathrm{BV}_{2}(\Omega)$ has a continuous representative. We emphasize this fact together with an estimate that will be often used in the following results. 
Lemma 2.1. Let $v \in \mathrm{BV}_{2}(\Omega)$. Then $v$ has a continuous representative (again denoted by $v$ ), and there exists a constant $c$ (not depending on $v$ or $\Omega$ ) such that for any ball $B(x, r) \subset \Omega$ the estimate

$$
\sup _{y \in B(x, r)}\left|v(y)-v(x)-(y-x) \cdot f_{B(x, r)} \nabla v(z) \mathrm{d} z\right| \leq c\left\|D^{2} v\right\|(B(x, r))
$$

holds.

Here and in the sequel, $B(x, r)$ denotes the open ball with center $x$ and radius $r$, $B(x, r)=\left\{z \in \mathbb{R}^{2}:|z-x|<r\right\}$.

Proof. The existence of a continuous representative for $v$ follows from Remark 2 of $\$ 1.4 .5$ in [28] (see also [35]). Because of coordinate invariance it is sufficient to prove the estimate $(2.1)$ for the case $\Omega=B(0,1)=B(x, r)$. Furthermore we may assume $v \in C^{\infty}(\Omega)$. By results of $\S 1.1 .15$ in [28] for any $u \in \mathrm{W}^{2,1}(\Omega)$ the estimate

$$
\sup _{y \in \Omega}|u(y)| \leq c(p)\left(p(u)+\left\|D^{2} u\right\|(\Omega)\right),
$$

holds, where $p(\cdot)$ is any continuous seminorm in $\mathrm{W}^{2,1}(\Omega)$ such that $p(g)=0 \Leftrightarrow$ $g=0$ for all first-order polynomials $g$. Clearly

$$
p(u)=|u(0)|+\left|f_{\Omega} \nabla u(z) \mathrm{d} z\right|
$$

is a continuous seminorm satisfying the above conditions. Now if we take

$$
u(y)=v(y)-v(0)-y \cdot f_{\Omega} \nabla v(z) \mathrm{d} z
$$

then $p(u)=0$ and the inequality (2.2) turns into the estimate (2.1).

In the sequel we shall always select the continuous representative when discussing $\mathrm{BV}_{2}$ functions.

In the following lemma, and for the remainder of the paper, we understand by an interval a closed square $I=[a, a+l] \times[b, b+l]$ with sides parallel to the coordinate axes. Furthermore we write $\ell(I)=l$ for its sidelength and $I^{\circ}=(a, a+l) \times(b, b+l)$ for its interior. Of course, the analog of the estimate (2.1) is valid if we replace the balls $B(x, r)$ by the corresponding intervals. In particular, we have:

Corollary 2.2. Let $v \in \mathrm{BV}_{2}(\Omega)$. Then for any interval $I=[a, a+l] \times[b, b+l] \subset \Omega$ the estimate

$$
\underset{I}{\operatorname{Osc}}(v) \leq C\left(\left\|D^{2} v\right\|\left(I^{\circ}\right)+\frac{1}{\ell(I)} \int_{I}|\nabla v|\right)
$$

holds, where $C$ does not depend on $v$ or $I$. 
By $\mathcal{L}^{k}(F)$ we denote the outer Lebesgue measure of a set $F \subset \mathbb{R}^{k}$. Denote by $\mathcal{H}^{1}$ and $\mathcal{H}_{\infty}^{1}$ the 1-dimensional Hausdorff measure, Hausdorff content, respectively: for any $F \subset \mathbb{R}^{k}, \mathcal{H}^{1}(F)=\lim _{\alpha \searrow 0} \mathcal{H}_{\alpha}^{1}(F)=\sup _{\alpha>0} \mathcal{H}_{\alpha}^{1}(F)$, where for each $0<\alpha \leq \infty$,

$$
\mathcal{H}_{\alpha}^{1}(F)=\inf \left\{\sum_{i=1}^{\infty} \operatorname{diam} F_{i}: \operatorname{diam} F_{i} \leq \alpha, \quad F \subset \bigcup_{i=1}^{\infty} F_{i}\right\} .
$$

It is well known that the equalities $\mathcal{H}^{1}(F)=\mathcal{H}_{\infty}^{1}(F)=\mathcal{L}^{1}(F)$ hold for any subset $F \subset \mathbb{R}$, whereas the set functions $\mathcal{H}^{1}$ and $\mathcal{H}_{\infty}^{1}$ are distinct in higher dimensions. For a Lebesgue measurable set $F \subset \mathbb{R}^{2}$ and a point $x \in \mathbb{R}^{2}$ we write

$$
\bar{D}(F, x)=\limsup _{r \rightarrow 0+} \frac{\mathcal{L}^{2}(F \cap B(x, r))}{\mathcal{L}^{2}(B(x, r))}, \quad \underline{D}(F, x)=\liminf _{r \rightarrow 0+} \frac{\mathcal{L}^{2}(F \cap B(x, r))}{\mathcal{L}^{2}(B(x, r))},
$$

$\operatorname{Int}_{M} F=\{x: \underline{D}(F, x)=1\}, \mathrm{Cl}_{M} F=\{x: \bar{D}(F, x)>0\}, \partial^{M} F=\mathrm{Cl}_{M} F \backslash \operatorname{Int}_{M} F$.

Finally recall that for any function $f \in \mathrm{BV}(U)$, where $U$ is an open set in $\mathbb{R}^{2}$, the coarea formula

$$
\|D f\|(U)=\int_{-\infty}^{+\infty} \mathcal{H}^{1}\left(U \cap \partial^{M}\{f \leq t\}\right) \mathrm{d} t
$$

holds (see for instance $\S 5.5$ in [15]).

\section{On images of sets of small capacities under $B V_{2}$ functions on the plane}

The main result of this section is the following Luzin $N$ property that we establish for $\mathrm{BV}_{2}$ functions:

Theorem 3.1. Let $v \in \mathrm{BV}_{2}\left(\mathbb{R}^{2}\right)$. Then for any $\varepsilon>0$ there exists $\delta>0$ such that for any set $E \subset \mathbb{R}^{2}$, if $\mathcal{H}_{\infty}^{1}(E)<\delta$ then $\mathcal{L}^{1}(v(E))<\varepsilon$.

Since balls are extension domains for $\mathrm{BV}_{2}$, Theorem 3.1 implies the following assertion:

Corollary 3.2. If $v \in \mathrm{BV}_{2, \mathrm{loc}}(\Omega), E \subset \Omega$ and $\mathcal{H}^{1}(E)=0$, then $\mathcal{L}^{1}(v(E))=0$.

For the remainder of this section we fix a function $v \in \mathrm{BV}_{2}\left(\mathbb{R}^{2}\right)$. To prove Theorem 3.1 we need some preliminary lemmas that we turn to next. The first is an immediate consequence of Corollary 2.2.

Corollary 3.3. For each interval $I \subset \mathbb{R}^{2}$ of sidelength $\ell(I)$ we have

$$
\mathcal{L}^{1}(v(I)) \leq C\left(\left\|D^{2} v\right\|\left(I^{\circ}\right)+\frac{1}{\ell(I)} \int_{I}|\nabla v|\right)
$$

where $C$ does not depend on $v$ or $I$. 
The total variation measure in (3.1) is estimated by use of the following:

Lemma 3.4. For any $\varepsilon>0$ there exists $\delta>0$ such that, for any open set $U \subset \mathbb{R}^{2}$, if $\mathcal{H}_{\infty}^{1}(U)<\delta$ then $\left\|D^{2} v\right\|(U)<\varepsilon$.

Proof. This is a consequence of the coarea formula and the following fact: if $F \subset \mathbb{R}^{2}$ is a Borel set with $\mathcal{H}^{1}(F)<\infty$, then for any decreasing sequence of open sets $U_{j} \supset U_{j+1}$ such that $\mathcal{H}_{\infty}^{1}\left(U_{j}\right) \rightarrow 0$ the convergence $\mathcal{H}^{1}\left(F \cap U_{j}\right) \rightarrow 0$ holds (see Theorem 1 (iv) of $\S 1.1 .1$ in [15]). We leave the details to the interested reader.

Lemma 3.5. For each $f \in \mathrm{BV}\left(\mathbb{R}^{2}\right)$ and for any $\varepsilon>0$ there exists a pair of functions $f_{0}, f_{1} \in \mathrm{BV}\left(\mathbb{R}^{2}\right)$ such that

$$
f=f_{0}+f_{1} \quad \text { with } \quad\left\|f_{0}\right\|_{\mathrm{L}^{\infty}} \leq K \text { and }\left\|f_{1}\right\|_{\mathrm{BV}}<\varepsilon,
$$

where $K=K(\varepsilon, f)$.

Proof. The proof is similar to the proof of Theorem 3 of $\S 5.9$ in [15]. Let $K>0$ and denote

$$
f_{0}(x)=\max \{\min \{f(x), K\},-K\}, \quad f_{1}(x)=f(x)-f_{0}(x) .
$$

Obviously $\left\|f_{1}\right\|_{\mathrm{L}^{1}}<\frac{1}{2} \varepsilon_{0}$ for sufficiently large $K$. By the lattice property of BV we have $f_{0}, f_{1} \in \mathrm{BV}\left(\mathbb{R}^{2}\right)$, and so we can use the coarea formula to compute

$$
\left\|D f_{1}\right\|\left(\mathbb{R}^{2}\right)=\int_{t:|t|>K} \mathcal{H}^{1}\left(\partial^{M}\{f \leq t\}\right) \mathrm{d} t .
$$

It follows that $\left\|f_{1}\right\|_{\mathrm{BV}}<\varepsilon_{0}$ for sufficiently large $K$.

We apply Lemma 3.5 componentwise to get:

Corollary 3.6. Let $v \in \mathrm{BV}_{2}\left(\mathbb{R}^{2}\right)$. For any $\varepsilon>0$ there exist vector functions $f_{0}$, $f_{1} \in \mathrm{BV}\left(\mathbb{R}^{2}, \mathbb{R}^{2}\right)$ such that

$$
\nabla v=f_{0}+f_{1} \quad \text { with } \quad\left\|f_{0}\right\|_{\mathrm{L}^{\infty}} \leq K \quad \text { and } \quad\left\|f_{1}\right\|_{\mathrm{BV}}<\varepsilon,
$$

where $K=K(\varepsilon, \nabla v)$.

The next result is an approximation result. Related results have appeared before in the literature, however, it appears that our result is somewhat more explicit.

Lemma 3.7 (see also [6]). Denote by $\mathcal{C}$ the collection of all functions of the form

$$
\varphi=\frac{1}{\mathcal{H}^{1}(\partial \Omega)} 1_{\Omega}
$$

where $\Omega$ is a bounded domain in $\mathbb{R}^{2}$ with a $\mathrm{C}^{\infty}$ smooth boundary $\partial \Omega$, and $1_{\Omega}$ its indicator function. If $f \in \mathrm{BV}\left(\mathbb{R}^{2}\right)$ and

$$
\|D f\|\left(\mathbb{R}^{2}\right) \leq 1,
$$


then there exists a sequence of functions $f_{n}: \mathbb{R}^{2} \rightarrow \mathbb{R}$ such that $f_{n} \rightarrow f$ pointwise almost everywhere and each function $f_{n}$ is a convex combination of functions from $\mathcal{C} \cup(-\mathcal{C})$.

Proof. We may assume without loss of generality that

$$
f \geq 0, \quad\|D f\|\left(\mathbb{R}^{2}\right)<1,
$$

see for instance the proof of Lemma 3.5. Since each function from $B V\left(\mathbb{R}^{2}\right)$ can be approximated strictly in $\mathrm{BV}$ by functions from $\mathrm{C}_{0}^{\infty}\left(\mathbb{R}^{2}\right)$ (see $\S 5.2 .2$ in [15]), we may also assume without loss of generality that

$$
f \in \mathrm{C}_{0}^{\infty}\left(\mathbb{R}^{2}\right), \quad \operatorname{supp} f \subset B(0, R), \quad f\left(\mathbb{R}^{2}\right) \subset[0, M) .
$$

By the classical Morse-Sard Theorem the set $D=\nabla f\left(\left\{x \in \mathbb{R}^{2}: \operatorname{det} \nabla^{2} f(x)=0\right\}\right)$ is $\mathcal{L}^{2}$ negligible, and hence we can in particular find $z \in \mathbb{R}^{2}$, arbitrarily close to 0 , such that $z \notin D$. Notice that then all critical points of $x \mapsto f(x)-z \cdot x$ are Morse regular: if $\nabla f(x)=z$ then $\operatorname{det} \nabla^{2} f(x) \neq 0$. It follows that the critical points for $f(x)-z \cdot x$ are isolated points in $B(0, R)$, and hence that there are at most finitely many. It is then clear that we can find $c \in \mathbb{R}$, arbitrarily close to 0 , such that all the critical values of $x \mapsto f(x)-z \cdot x-c$ are irrational numbers.

Thus by considering perturbations of the above form we find, for a given $\delta \in$ $(0,1), \mathrm{C}^{\infty}$ functions $f_{\delta}$ satisfying the three conditions:

(i)

$$
\left\|\nabla f_{\delta}\right\|_{\mathrm{L}^{1}(B(0, R))}<1
$$

$$
\sup _{x \in B(0, R)}\left|f(x)-f_{\delta}(x)\right|<\delta, \quad\left\|\nabla f-\nabla f_{\delta}\right\|_{\mathrm{L}^{1}(B(0, R))}<\delta .
$$

(iii) All the critical values of the function $f_{\delta}$ are irrational numbers and they are regular in the sense of Morse theory.

Let $t>\delta$ be a rational number. Then by (iii), (3.5), and the Implicit Function Theorem we can decompose the preimage as

$$
\left\{x \in B(0, R): f_{\delta}(x)>t\right\}=\bigcup_{i=1}^{m_{t}} \Omega_{i}
$$

where $m_{t} \in \mathbb{N}$, each $\Omega_{i}=\Omega_{i}^{t}$ is a bounded $\mathrm{C}^{\infty}$ smooth domain, and

$$
\bigcup_{i=1}^{m_{t}} \partial \Omega_{i}=\left\{x \in B(0, R): f_{\delta}(x)=t\right\}, \quad\left(\mathrm{Cl} \Omega_{i}\right) \cap \mathrm{Cl} \Omega_{j}=\emptyset, \quad \mathrm{Cl} \Omega_{i} \subset B(0, R)
$$

for $1 \leq i, j \leq m_{t}$ and $i \neq j$. We remark that $m_{t}<\infty$ since $\nabla f_{\delta} \neq 0$ on the level set $\left\{x \in B(0, R): f_{\delta}(x)=t\right\}$.

Next we define the function $h:[\delta, M] \rightarrow \mathbb{R}$ by the formula

$$
h(t)=\mathcal{H}^{1}\left(\left\{x \in B(0, R): f_{\delta}(x)=t\right\}\right) .
$$


It is easy to check (by elementary calculus) that $h(t)$ is continuous on $[\delta, M]$ (because of our assumption (iii)). In particular, the function $h$ is Riemann integrable on $[\delta, M]$, so that with $t_{j}=\frac{j}{k}, J_{k}=\{j \in \mathbb{N}: k \delta<j<k M\}$ we have convergence of the Riemann sums:

$$
\sum_{j \in J_{k}} \frac{1}{k} h\left(t_{j}\right) \rightarrow \int_{\delta}^{M} h(t) \mathrm{d} t \quad \text { as } k \rightarrow \infty .
$$

By (i) and the coarea formula,

$$
\int_{\delta}^{M} h(t) \mathrm{d} t<1
$$

so we may take $k \in \mathbb{N}$ so large that $k>2 / \delta$ and

$$
\sum_{j \in J_{k}} \frac{1}{k} h\left(t_{j}\right)<1
$$

We fix such a value for $k$, and write $m_{t_{j}}=m_{j}, \Omega_{i}^{t_{j}}=\Omega_{i}^{j}$ and

$$
\left\{x \in B(0, R): f_{\delta}(x)>t_{j}\right\}=\bigcup_{i=1}^{m_{j}} \Omega_{i}^{j},
$$

where $\Omega_{i}^{t_{j}}$ are the sets described above. Put

$$
f_{k}=\sum_{j \in J_{k}} \sum_{i=1}^{m_{j}} \frac{1}{k} 1_{\Omega_{i}^{j}}
$$

and note that by construction

$$
\left\|f-f_{k}\right\|_{L^{\infty}(B(0, R))}<3 \delta+\frac{2}{k}<4 \delta .
$$

Finally we write

$$
f_{k}=\sum_{j \in J_{k}} \sum_{i=1}^{m_{j}} \alpha_{i j} \frac{1_{\Omega_{j}^{i}}}{\mathcal{H}^{1}\left(\partial \Omega_{j}^{i}\right)}, \quad \text { with } \quad \alpha_{i j}=\frac{\mathcal{H}^{1}\left(\partial \Omega_{j}^{i}\right)}{k},
$$

where, by (3.6) and (3.7),

$$
\sum_{j \in J_{k}} \sum_{i=1}^{m_{j}} \alpha_{i j}<1
$$

From (ii), (3.8) and (3.9) we arrive at the required assertion.

Definition 3.8. Let $\mu$ be a positive measure on $\mathbb{R}^{2}$. We say that $\mu$ has property $(*)$ if $\mu$ is absolutely continuous with respect to Lebesgue measure and

$$
\mu(I) \leq \ell(I)
$$

for any interval $I \subset \mathbb{R}^{2}$. 
The following result could also be deduced from Theorem 5.12.4 in [39] and from $\$ 1.4 .3$ in [28], but for the convenience of the reader we give an elementary direct proof based on Lemma 3.7 .

Lemma 3.9. If $f \in \mathrm{BV}\left(\mathbb{R}^{2}\right)$ and $\mu$ has property $(*)$, then

$$
\left|\int f d \mu\right| \leq C\|D f\|\left(\mathbb{R}^{2}\right)
$$

where $C$ does not depend on $\mu$ or $f$.

Proof. In view of Lemma 3.7 and the Fatou lemma (note that $\mu$ is absolutely continuous with respect to Lebesgue measure), it is sufficient to bound $\int \varphi \mathrm{d} \mu$ for functions of the special form

$$
\varphi=\frac{1}{\mathcal{H}^{1}(\partial \Omega)} 1_{\Omega},
$$

where $\Omega$ is a bounded domain in $\mathbb{R}^{2}$ with a smooth boundary $\partial \Omega$. Obviously $\Omega \subset I$, where $I$ is an interval with sidelength $\ell(I) \sim \operatorname{diam} \Omega \leq \mathcal{H}^{1}(\partial \Omega)$. Hence from property $(*)$,

$$
\int \varphi \mathrm{d} \mu \leq \frac{\mu(I)}{\mathcal{H}^{1}(\partial \Omega)} \lesssim \frac{\mu(I)}{\ell(I)}<C,
$$

as required.

Since $|\nabla f| \in \mathrm{BV}\left(\mathbb{R}^{2}\right)$ and $\|D|\nabla f|\| \leq\left\|D^{2} f\right\|$ as measures when $f \in \mathrm{BV}_{2}\left(\mathbb{R}^{2}\right)$, we infer the

Corollary 3.10. If $f \in \mathrm{BV}_{2}\left(\mathbb{R}^{2}\right)$ and $\mu$ is a measure with property $(*)$, then

$$
\int|\nabla f| \mathrm{d} \mu \leq C\left\|D^{2} f\right\|\left(\mathbb{R}^{2}\right),
$$

where $C$ does not depend on $\mu$ or $f$.

By a dyadic interval we understand a square of the form $\left[\frac{k}{2^{m}}, \frac{k+1}{2^{m}}\right] \times\left[\frac{l}{2^{m}}, \frac{l+1}{2^{m}}\right]$, where $k, l$ and $m$ are integers. The following assertion is straightforward, and hence we omit its proof here.

Lemma 3.11. For any bounded set $F \subset \mathbb{R}^{2}$ there exist dyadic intervals $I_{1}, \ldots, I_{4}$ such that $F \subset I_{1} \cup \cdots \cup I_{4}$ and $\ell\left(I_{1}\right)=\cdots=\ell\left(I_{4}\right) \leq 2 \operatorname{diam} F$.

Proof of Theorem 3.1. Fix $\varepsilon>0$ and let $E \subset \mathbb{R}^{2}$ be a set with $\mathcal{H}_{\infty}^{1}(E)<\delta$, where $\delta>0$ will be specified below. By virtue of Corollary 3.6 we can find a decomposition $\nabla v=f_{0}+f_{1}$, where $\left\|f_{0}\right\|_{\mathrm{L}^{\infty}} \leq K=K(\varepsilon, \nabla v)$ and $\left\|f_{1}\right\|_{\mathrm{BV}}<\varepsilon$. In view of Lemma 3.11 we can find a collection $\left\{I_{\alpha}\right\}$ of dyadic intervals satisfying

$$
E \subset \bigcup I_{\alpha}
$$


and

$$
\sum_{\alpha} \ell\left(I_{\alpha}\right)<16 \delta<\frac{1}{K+1} \varepsilon
$$

where we imposed our first condition on $\delta$. Define

$$
\mathcal{F}=\left\{J: J \subset \mathbb{R}^{2} \text { dyadic interval; } \sum_{I_{\alpha} \subset J} \ell\left(I_{\alpha}\right) \geq \ell(J)\right\} .
$$

Thus $I_{\alpha} \in \mathcal{F}$ for each $\alpha$. Denote by $\mathcal{F}^{*}=\left\{J_{\beta}\right\}$ the collection of maximal elements of $\mathcal{F}$. Clearly

$$
E \subset \bigcup_{\alpha} I_{\alpha} \subset \bigcup_{\beta} J_{\beta},
$$

and since dyadic intervals are either disjoint or contained in one another, the $\left\{J_{\beta}\right\}$ are mutually disjoint. It follows that

$$
\sum_{\beta} \ell\left(J_{\beta}\right) \leq \sum_{\beta} \sum_{I_{\alpha} \subset J_{\beta}} \ell\left(I_{\alpha}\right) \leq \sum_{\alpha} \ell\left(I_{\alpha}\right)<\frac{1}{K+1} \varepsilon .
$$

Observe also that for any dyadic interval $Q \subset \mathbb{R}^{2}$,

$$
\sum_{J_{\beta} \subset Q} \ell\left(J_{\beta}\right) \leq \sum_{I_{\alpha} \subset Q} \ell\left(I_{\alpha}\right) \leq 2 \ell(Q) .
$$

We used here that if $J_{\beta} \subset Q$ for some $\beta$, then either $J_{\beta}=Q$ or $Q \notin \mathcal{F}$ (because $J_{\beta}$ is maximal); in both cases (3.16) holds. Define the measure $\mu$ by

$$
\mu=\left(\sum_{\beta} \frac{1}{\ell\left(J_{\beta}\right)} 1_{J_{\beta}}\right) \mathcal{L}^{2} .
$$

Claim. $\frac{1}{48} \mu$ has property $(*)$.

Indeed, for a dyadic interval $Q$, write

$$
\mu(Q)=\sum_{J_{\beta} \subset Q} \ell\left(J_{\beta}\right)+\sum_{Q \subset J_{\beta}} \frac{\ell(Q)^{2}}{\ell\left(J_{\beta}\right)} \leq 3 \ell(Q),
$$

where we have used (3.16) and the fact that $Q \subset J_{\beta}$ for at most one $\beta$. Then for any interval $I$ we have the estimate $\mu(I) \leq 48 \ell(I)$ (see Lemma 3.11). This proves the claim.

Now return to $\mathcal{L}^{1}(v(E))$. From $(3.14)$ we get

$$
v(E) \subset \bigcup_{\beta} v\left(J_{\beta}\right) .
$$

In addition to the condition in (3.13) we now decrease $\delta>0$ further so that, using Lemma 3.4 and inequality (3.15), we may assume

$$
\sum_{\beta}\left\|D^{2} v\right\|\left(J_{\beta}^{\circ}\right)<\varepsilon .
$$


By Corollaries 3.3, 3.6 and 3.9 we estimate as follows:

$$
\begin{aligned}
\sum_{\beta} \mathcal{L}^{1}\left(v\left(J_{\beta}\right)\right) & \leq C \sum_{\beta}\left\|D^{2} v\right\|\left(J_{\beta}^{\circ}\right)+C \sum_{\beta} \frac{1}{\ell\left(J_{\beta}\right)} \int_{J_{\beta}}|\nabla v| \\
& \leq C \varepsilon+C \frac{K}{K+1} \varepsilon+C \sum_{\beta} \frac{1}{\ell\left(J_{\beta}\right)} \int_{J_{\beta}}\left|f_{1}\right| \\
& =C^{\prime} \varepsilon+C \int\left|f_{1}\right| d \mu \leq C^{\prime \prime} \varepsilon
\end{aligned}
$$

Since $\varepsilon>0$ was arbitrary, the proof of Theorem 3.1 is complete.

\section{Morse-Sard theorem in $\mathrm{BV}_{2}$}

Before stating the main result of this section we shall define our notion of critical set for a function $v \in \mathrm{BV}_{2 \text {,loc }}(\Omega)$, where $\Omega \subset \mathbb{R}^{2}$ is an open set. First we let for $\varepsilon>0$,

$$
E_{\varepsilon}=\{x \in \Omega:|\nabla v(x)| \leq \varepsilon\},
$$

and note that $\mathrm{Cl}_{M} E_{\varepsilon}$ does not depend on the particular representative we use for $\nabla v$ when defining $E_{\varepsilon}$. Define

$$
Z_{0 v}=\Omega \cap\left(\bigcap_{\varepsilon>0} \mathrm{Cl}_{M} E_{\varepsilon}\right),
$$

and

$$
Z_{1 v}=\left\{x \in \Omega: v \text { is differentiable at } x \text { and } v^{\prime}(x)=0\right\},
$$

where in $Z_{1 v}$ we refer to the continuous representative of $v$ (see also Lemma 4.2 below). The critical set for $v$ is the union $Z_{v}=Z_{0 v} \cup Z_{1 v}$.

Theorem 4.1. Suppose $v \in \mathrm{BV}_{2, \text { loc }}(\Omega)$, where $\Omega$ is an open subset of $\mathbb{R}^{2}$. Then $\mathcal{L}^{1}\left(v\left(Z_{v}\right)\right)=0$.

The proof of Theorem 4.1 splits into a number of lemmas. We require the following result due to Dorronsoro [13] about differentiability properties of $\mathrm{BV}_{2}$ functions.

Lemma 4.2 (see [13], Theorems B and 1). Suppose that $v \in \mathrm{BV}_{2, \mathrm{loc}}(\Omega)$, where $\Omega$ is an open subset of $\mathbb{R}^{2}$. Then we can choose a Borel representative of $\nabla v$ such that there exist a decomposition $\Omega=K_{v} \cup G_{v} \cup A_{v}$ and mappings $\lambda: \Omega \rightarrow \mathbb{R}^{2}$, $\mu: \Omega \rightarrow \mathbb{R}^{2}$, and $\nu: K_{v} \rightarrow \mathbb{S}^{1}$ with the following properties:

(i) $\mathcal{H}^{1}\left(A_{v}\right)=0$.

(ii) $K_{v}=\bigcup_{i} K_{i}$ as an at most countable disjoint union, where each $K_{i}$ is a compact subset of some $\mathrm{C}^{1}$ curve $L_{i}$. Moreover, $\nu(x)$ is perpendicular to $L_{i}$ at $x$ if $x \in K_{i}$. 
(iii) For all $x \in G_{v}, \nabla v(x)=\lambda(x)=\mu(x)$ and, as $r \searrow 0$,

$$
f_{B(x, r)}|\nabla v(z)-\nabla v(x)|^{2} \mathrm{~d} z \rightarrow 0, \quad \sup _{y \in B(x, r)} r^{-1}|v(y)-v(x)-(y-x) \cdot \nabla v(x)| \rightarrow 0,
$$

and hence $v$ is in particular differentiable at $x$.

(iv) For all $x \in K_{v}$,

$$
\begin{gathered}
\lim _{r \searrow 0} f_{B_{+}(x, r)}|\nabla v(z)-\lambda(x)|^{2} \mathrm{~d} z=0, \quad \lim _{r \searrow 0} f_{B_{-}(x, r)}|\nabla v(z)-\mu(x)|^{2} \mathrm{~d} z=0, \\
\sup _{y \in B_{+}(x, r)} r^{-1}|v(y)-v(x)-(y-x) \cdot \lambda(x)| \rightarrow 0 \quad \text { as } r \searrow 0, \\
\sup _{y \in B_{-}(x, r)} r^{-1}|v(y)-v(x)-(y-x) \cdot \mu(x)| \rightarrow 0 \quad \text { as } r \searrow 0,
\end{gathered}
$$

where

$$
\begin{aligned}
& B_{+}(x, r)=\{y \in B(x, r):(y-x) \cdot \nu(x)>0\}, \\
& B_{-}(x, r)=\{y \in B(x, r):(y-x) \cdot \nu(x)<0\} .
\end{aligned}
$$

Observe that with our definition of the critical set $Z_{v}$ the following inclusion holds:

$$
Z_{v} \supset\left\{x \in G_{v}: \nabla v(x)=0\right\} \cup\left\{x \in K_{v}: \mu(x)=0 \text { or } \lambda(x)=0\right\} .
$$

The next result, which is due to Ambrosio, Caselles, Masnou and Morel, concerns a measure theoretic notion of connectedness for sets of finite perimeter. In its statement we write $A=B\left(\bmod \mathcal{H}^{1}\right)$ for two subsets $A, B \subset \mathbb{R}^{2}$ when their symmetric difference is $\mathcal{H}^{1}$-negligible, that is, when $\mathcal{H}^{1}((A \backslash B) \cup(B \backslash A))=0$.

Lemma 4.3 ([3]). For any Lebesgue measurable set $F \subset \mathbb{R}^{2}$ with $\mathcal{H}^{1}\left(\partial^{M} F\right)<\infty$ there is a finite or countable family $\left\{F_{i}\right\}_{i \in I}$ and a set $T \subset \mathbb{R}^{2}$ with the following properties:

(i) The $F_{i}$ are measurable sets, $\mathcal{L}^{2}\left(F_{i}\right)>0, \mathcal{H}^{1}\left(\partial^{M} F_{i}\right)<\infty$.

(ii) $F=\bigcup_{i \in I} F_{i}$, and $F_{i} \cap F_{j}=\emptyset$ for $i \neq j$.

(iii) $\left(\partial^{M} F_{i}\right) \cap\left(\partial^{M} F_{j}\right)=\emptyset\left(\bmod \mathcal{H}^{1}\right)$ for $i \neq j$.

(iv) $\partial^{M} F=\bigcup_{i \in I} \partial^{M} F_{i}\left(\bmod \mathcal{H}^{1}\right)$, so in particular, $\mathcal{H}^{1}\left(\partial^{M} F\right)=\sum_{i \in I} \mathcal{H}^{1}\left(\partial^{M} F_{i}\right)$.

(v) $\mathcal{H}^{1}\left(\operatorname{Int}_{M} F \backslash\left(\bigcup_{i \in I} \operatorname{Int}_{M} F_{i}\right)\right)=0$.

(vi) $\mathcal{H}^{1}(T)=0$.

(vii) For any set $L$ with $\mathcal{H}^{1}(L)=0$ and for any $x, y \in \operatorname{Int}_{M} F_{i} \backslash(T \cup L)$ and $\delta>0$ there exists a rectifiable curve $\Gamma \subset\left(\operatorname{Int}_{M} F_{i}\right) \backslash(T \cup L)$ joining $x$ to y so that

$$
\mathcal{H}^{1}(\Gamma) \leq|x-y|+\mathcal{H}^{1}\left(\partial^{M} F_{i}\right)+\delta .
$$

Proof. See Proposition 3, Theorems 1 and 8 (together with the subsequent remark) from $[3]$. 
Lemma 4.4. If the set $F$ in Lemma 4.3 is bounded, then we can reformulate the property (vii) in the following way:

(vii') for any set $L$ with $\mathcal{H}^{1}(L)=0$ and for any $x, y \in\left(\operatorname{Int}_{M} F_{i}\right) \backslash(T \cup L)$ and $\delta>0$ there exists a rectifiable curve $\Gamma \subset\left(\operatorname{Int}_{M} F_{i}\right) \backslash(T \cup L)$ joining $x$ to $y$ so that

$$
\mathcal{H}^{1}(\Gamma) \leq 2 \mathcal{H}^{1}\left(\partial^{M} F_{i}\right)+\delta .
$$

Proof. See Lemma 4.2 in [32].

Since the assertion of Theorem 4.1 has a local nature, for the remainder of the section we may assume without loss of generality that $\Omega=B(0,1)$ and $v \in \mathrm{BV}_{2}(\Omega)$. Moreover, because of the Sobolev Extension Theorem we may assume that $v$ is defined on all of $\mathbb{R}^{2}$ and $v \in \mathrm{BV}_{2}\left(\mathbb{R}^{2}\right)$. (However, we will calculate the critical set $Z_{v}$ and the corresponding sets $E_{\varepsilon}$ by the above formulas with respect to $\Omega=B(0,1)$.)

Lemma 4.5. Suppose $\mathcal{H}^{1}\left(\partial^{M} E_{\varepsilon}\right)<\infty$. Let $E_{\varepsilon}^{i}$ be the sets from Lemmas $4.3-4.4$ applying to $F=E_{\varepsilon}$. Then $\operatorname{diam}\left(v\left(\mathrm{Cl}_{M} E_{\varepsilon}^{i}\right)\right) \leq 2 \varepsilon \mathcal{H}^{1}\left(\partial^{M} E_{\varepsilon}^{i}\right)$.

Proof. The proof is based on Lemmas 4.2 and 4.4. First we apply Lemma 4.4 with $F=E_{\varepsilon} \subset B(0,1)$ and take $L=A_{v}$, where $A_{v}$ is the set defined in Lemma 4.2. Accordingly, given $\delta>0$ and points $x, y \in \operatorname{Int}_{M} E_{\varepsilon}^{i} \backslash\left(T \cup A_{v}\right)$ we can find a rectifiable curve $\Gamma \subset \operatorname{Int}_{M} E_{\varepsilon}^{i} \backslash\left(T \cup A_{v}\right)$ joining $x$ to $y$ with

$$
\mathcal{H}^{1}(\Gamma) \leq 2 \mathcal{H}^{1}\left(\partial^{M} E_{\varepsilon}^{i}\right)+\delta .
$$

Now $\Gamma \cap A_{v}=\emptyset$, so $\Gamma \subset G_{v} \cup K_{v}$ by Lemma 4.2. If $z \in G_{v} \cap \operatorname{Int}_{M} E_{\varepsilon}^{i}$, then $v$ is differentiable at $z$ and $|\nabla v(z)| \leq \varepsilon$. If $z \in K_{v} \cap \operatorname{Int}_{M} E_{\varepsilon}^{i}$, then we check that the mappings $\lambda, \mu$ defined in Lemma 4.2 satisfy $|\lambda(z)|,|\mu(z)| \leq \varepsilon$. From (iii) and (iv) of Lemma 4.2, we deduce that the restriction $\left.v\right|_{\Gamma}$ is $\varepsilon$-Lipschitz, and hence

$$
|v(x)-v(y)| \leq 2 \varepsilon \mathcal{H}^{1}\left(\partial^{M} E_{\varepsilon}^{i}\right)+\varepsilon \delta .
$$

Because $T \cup A_{v}$ is negligible, $\left(\operatorname{Int}_{M} E_{\varepsilon}^{i}\right) \backslash\left(T \cup A_{v}\right)$ is dense $\operatorname{in} \operatorname{Int}_{M} E_{\varepsilon}^{i}$, and using that almost all points are density points we conclude that $\mathrm{Cl}\left(\operatorname{Int}_{M} E_{\varepsilon}^{i} \backslash\left(T \cup A_{v}\right)\right) \supset$ $\mathrm{Cl}_{M} E_{\varepsilon}^{i}$. Since $v$ is continuous, (4.1) then easily yields the assertion of the lemma.

Lemma 4.6. For any $\varepsilon>0$ the inequality $\mathcal{H}^{1}\left(v\left(\mathrm{Cl}_{M} E_{\varepsilon}\right)\right) \leq 2 \varepsilon \mathcal{H}^{1}\left(\partial^{M} E_{\varepsilon}\right)$ holds.

Proof. Suppose $\mathcal{H}^{1}\left(\partial^{M} E_{\varepsilon}\right)<\infty$. From properties (iv)-(v) of Lemma 4.3 we have $\mathrm{Cl}_{M} E_{\varepsilon}=\bigcup_{i \in I} \mathrm{Cl}_{M} E_{\varepsilon}^{i}\left(\bmod \mathcal{H}^{1}\right)$. So from Corollary 3.2 we obtain

$$
\mathcal{H}^{1}\left(v\left(\mathrm{Cl}_{M} E_{\varepsilon}\right)\right) \leq \sum_{i \in I} \mathcal{H}^{1}\left(v\left(\mathrm{Cl}_{M} E_{\varepsilon}^{i}\right)\right) \leq 2 \varepsilon \sum_{i \in I} \mathcal{H}^{1}\left(\partial^{M} E_{\varepsilon}^{i}\right)=2 \varepsilon \mathcal{H}^{1}\left(\partial^{M} E_{\varepsilon}\right),
$$

where the last equality follows from property (iv) of Lemma 4.3. 
Corollary 4.7. For any $\varepsilon>0$ we have the estimate

$$
\mathcal{H}^{1}\left(v\left(\mathrm{Cl}_{M} E_{\varepsilon}\right)\right) \leq 2 \varepsilon\left[\mathcal{H}^{1}\left(B(0,1) \cap \partial^{M} E_{\varepsilon}\right)+\mathcal{H}^{1}(\partial B(0,1))\right] .
$$

Corollary 4.8. The convergence

$$
\mathcal{H}^{1}\left(v\left(\mathrm{Cl}_{M} E_{\varepsilon}\right)\right) \rightarrow 0 \quad \text { as } \varepsilon \searrow 0
$$

holds.

Proof. This follows from Corollary 4.7 and the coarea formula (see also the proof of Proposition 4.3 in [32]).

Obviously the last corollary, together with Lemma 4.2 and Corollary 3.2, imply the statement of Theorem 4.1.

\section{Application to the level sets of $\mathrm{W}^{2,1}$ functions}

By a cycle we mean a set which is homeomorphic to the unit circle $\mathbb{S}^{1}$. The purpose of this section is to prove the following result:

Theorem 5.1. Suppose $v \in \mathrm{W}^{2,1}\left(\mathbb{R}^{2}\right)$. Then for almost all $y \in \mathbb{R}$ the preimage $v^{-1}(y)$ is a finite disjoint family of $\mathrm{C}^{1}$ cycles $S_{j}, j=1, \ldots, N(y)$. Moreover, the tangent vector to each $S_{j}$ is an absolutely continuous function of the natural parameter of $S_{j}$.

This means, in particular, that for each $S_{j}$ there exists a $\mathrm{C}^{1}$ diffeomorphism $\gamma: \mathbb{S}^{1} \ni s \mapsto \gamma(s) \in S_{j}$. Further, the last assertion of the theorem means that the components of the tangent vector to $S_{j}$ (more precisely, the components of $\nabla v$ ) are absolute continuous functions of the variable $s$.

Invoking extension theorems for Sobolev functions (see, for example, [28] and the references therein), we obtain the following:

Corollary 5.2. Suppose $\Omega \subset \mathbb{R}^{2}$ is a bounded domain with a Lipschitz boundary and $v \in \mathrm{W}^{2,1}(\Omega)$. Then for almost all $y \in \mathbb{R}$ the preimage $v^{-1}(y)$ is a finite disjoint family of $\mathrm{C}^{1}$ curves $\Gamma_{j}, j=1, \ldots, N(y)$. Each $\Gamma_{j}$ is a cycle in $\Omega$ or it is a simple arc with endpoints on $\partial \Omega$ (in the latter case, $\Gamma_{j}$ is transverse to $\partial \Omega$ ). Moreover, the tangent vector to each $\Gamma_{j}$ is an absolutely continuous function of the natural parameter of $\Gamma_{j}$.

For the remainder of the section we fix a function $v \in \mathrm{W}^{2,1}\left(\mathbb{R}^{2}\right)$. Now the set $K_{v}$ from Lemma 4.2 is empty (since $\nabla v \in \mathrm{W}^{1,1}$ and $\mathrm{W}^{1,1}$ mappings cannot have jump discontinuities, see also the proofs in [13]). We therefore have the following result:

Lemma 5.3 (see also Theorem 1 of $\S 4.8$ in [15]). We can choose a Borel representative of $\nabla v$ such that there exists a set $A_{v} \subset \mathbb{R}^{2}$ with the following properties:

(i) $\mathcal{H}^{1}\left(A_{v}\right)=0$. 
(ii) For each fixed $x \in \mathbb{R}^{2} \backslash A_{v}$ we have as $r \searrow 0$,

$$
f_{B(x, r)}|\nabla v(z)-\nabla v(x)|^{2} \mathrm{~d} z \rightarrow 0, \quad \sup _{y \in B(x, r)} r^{-1}|v(y)-v(x)-(y-x) \cdot \nabla v(x)| \rightarrow 0,
$$

and hence $v$ is in particular differentiable at $x$.

(iii) For any $\varepsilon>0$ there exists an open set $U \subset \mathbb{R}^{2}$ such that $\operatorname{Cap}_{1}(U)<\varepsilon$, $A_{v} \subset U$, and $\nabla v$ is continuous relative to $\mathbb{R}^{2} \backslash U$.

Further we fix the above representative of $\nabla v$. Here (see, for example, $\S 4.7$ in [15]) $\mathrm{Cap}_{1}$ denotes the 1-capacity defined for any set $E \subset \mathbb{R}^{2}$ as

$$
\operatorname{Cap}_{1}(E)=\inf _{f}\|\nabla f\|_{L^{1}}
$$

where the infimum is taken over all $f \in L^{2}\left(\mathbb{R}^{2}\right)$ with $D f \in L^{1}\left(\mathbb{R}^{2}\right)$ and so that $f \geq 1$ almost everywhere in an open neighborhood of $E$. The 1-capacity has the following known simple description.

Lemma 5.4 (see the proof of Theorem 3 from $\S 5.6 .3$ in [15]). There is a constant $C_{0}>0$ such that for any set $E \subset \mathbb{R}^{2}$ the following inequalities hold:

$$
\frac{1}{C_{0}} \mathcal{H}_{\infty}^{1}(E) \leq \operatorname{Cap}_{1}(E) \leq C_{0} \mathcal{H}_{\infty}^{1}(E)
$$

Lemma 5.5. For any $\varepsilon>0$ there exists an open set $U \subset \mathbb{R}^{2}$ and a function $g \in C^{1}\left(\mathbb{R}^{2}\right)$ such that $\operatorname{Cap}_{1}(U)<\varepsilon, A_{v} \subset U$ and $v=g, \nabla v=\nabla g$ on $\mathbb{R}^{2} \backslash U$.

Proof. Denote

$$
A_{\delta, \rho}=\left\{x \in \mathbb{R}^{n}: \exists r \in(0, \rho] \text { so that } \frac{1}{r}\left\|D^{2} v\right\|(B(x, r)) \geq \delta\right\} .
$$

Using Vitali's covering theorem and that $\left\|D^{2} v\right\|$ is absolutely continuous with respect to $\mathcal{L}^{2}$ (recall that $v$ is $\mathrm{W}^{2,1}$ ) it is easy to prove that for each fixed $\delta>0$,

$$
\operatorname{Cap}_{1}\left(A_{\delta, \rho}\right) \rightarrow 0 \text { as } \rho \searrow 0 \text {. }
$$

So we can choose a sequence $\rho_{j}>0$ such that

$$
\operatorname{Cap}_{1}\left(A_{\frac{1}{j}, \rho_{j}}\right) \leq 2^{-j}
$$

holds. Denoting

$$
A_{k}=\bigcup_{j \geq k} A_{\frac{1}{j}, \rho_{j}}
$$

we have

$$
\operatorname{Cap}_{1}\left(A_{k}\right) \leq 2^{-k+1}
$$

and for all $k \in \mathbb{N}, \alpha>0$ there exists $r_{k, \alpha}>0$ such that for all $x \in \mathbb{R}^{2} \backslash A_{k}$, $r \in\left(0, r_{k, \alpha}\right)$ we have

$$
\frac{1}{r}\left\|D^{2} v\right\|(B(x, r))<\alpha .
$$


It follows from the proof of Theorem 1 from $\S 4.8$ in [15] that there exists a sequence of mappings $f_{i} \in \mathrm{C}_{0}^{\infty}\left(\mathbb{R}^{2}, \mathbb{R}^{2}\right)$ such that for the sets

$$
B_{i}=\left\{x \in \mathbb{R}^{n}: \exists r>0 f_{B(x, r)}\left|\nabla v(z)-f_{i}(z)\right| \mathrm{d} z>2^{-i}\right\}, \quad F_{k}=A_{v} \cup\left(\bigcup_{j=k}^{\infty} B_{j}\right),
$$

where $A_{v}$ is the set from Lemma 5.3, we have

$$
\mathrm{Cap}_{1}\left(F_{k}\right) \rightarrow 0 \text { as } k \rightarrow \infty,
$$

and for all $x \in \mathbb{R}^{2} \backslash F_{k}$ and $i \geq k$,

$$
\left|f_{i}(x)-\nabla v(x)\right| \leq 2^{-i}
$$

From the above formulas, by direct calculation for all $x \in \mathbb{R}^{2} \backslash F_{k}, \quad i \geq k$, and $r>0$ we have

$$
\begin{aligned}
\left|\nabla v(x)-f_{B(x, r)} \nabla v(z) \mathrm{d} z\right| & \leq\left|\nabla v(x)-f_{i}(x)\right|+f_{B(x, r)}\left|\nabla v(z)-f_{i}(x)\right| \mathrm{d} z \\
& \leq 2^{-i+1}+\omega_{f_{i}}(r)
\end{aligned}
$$

where $\omega_{f_{i}}(r)=\max _{|z-x| \leq r}\left|f_{i}(z)-f_{i}(x)\right|$ is the modulus of continuity of $f_{i}$. Take a sequence of open sets $\bar{U}_{k} \supset F_{k} \cup A_{k}$ such that

$$
\mathrm{Cap}_{1} U_{k} \rightarrow 0 \quad \text { as } k \rightarrow \infty \text {. }
$$

Then from the formulas (5.4), (5.6)-(5.7) and Lemma 2.1 we obtain that there exists a function $\omega:(0,+\infty) \rightarrow(0,+\infty)$ such that $\omega(\delta) \rightarrow 0$ as $\delta \searrow 0$ and for all $k \in \mathbb{N}$ and for any pair $x, y \in \mathbb{R}^{2} \backslash U_{k}$ the estimates

$$
\begin{gathered}
|v(x)-v(y)| \leq \omega(|x-y|), \\
|\nabla v(x)-\nabla v(y)| \leq \omega(|x-y|), \\
|v(y)-v(x)-(y-x) \cdot \nabla v(x)| \leq \omega(|x-y|)|x-y|
\end{gathered}
$$

hold. Then the assertion of Lemma 5.5 follows from the last estimates, the convergence (5.8), and from the classical Whitney extension theorem (see, for example, Theorem 1 of $\S 6.5$ in $[15])$.

Using Theorems 3.1 and 4.1, and Lemma 5.4, we can reformulate the last lemma in the following way:

Corollary 5.6. For any $\varepsilon>0$ there exist an open set $V \subset \mathbb{R}$ and a function $g \in \mathrm{C}^{1}\left(\mathbb{R}^{2}\right)$ such that $\mathcal{H}_{\infty}^{1}(V)<\varepsilon, v\left(A_{v}\right) \subset V$, and $v=g, \nabla v=\nabla g \neq 0$ on $v^{-1}(\mathbb{R} \backslash V)$.

The inclusion $v \in \mathrm{W}^{2,1}\left(\mathbb{R}^{2}\right)$ and Corollary 2.2 easily imply the following statement:

Lemma 5.7. For any $\varepsilon>0$ there exists $R_{\varepsilon} \in(0,+\infty)$ such that $|v(x)|<\varepsilon$ for all $x \in \mathbb{R}^{2} \backslash B\left(0, R_{\varepsilon}\right)$. 
Proof of Theorem 5.1. Fix arbitrary $\varepsilon>0$. Take the corresponding set $V$ and the function $g \in \mathrm{C}^{1}\left(\mathbb{R}^{2}\right)$ from Corollary 5.6. Let $0 \neq y \in v\left(\mathbb{R}^{2}\right) \backslash V$. Denote $F_{v}=v^{-1}(y)$ and $F_{g}=g^{-1}(y)$. We assert the following properties of these sets:

(i) $F_{v}$ is a compact set;

(ii) $F_{v} \subset F_{g}$;

(iii) $\nabla v=\nabla g \neq 0$ on $F_{v}$;

(iv) The function $v$ is differentiable (in the classical sense) at each $x \in F_{v}$, and the classical derivative coincides with $\nabla v(x)$.

Indeed, (i) follows from Lemma 5.7, (ii)-(iii) follow from Corollary 5.6, and (iv) follows from Lemma 5.3 and from the condition $v\left(A_{v}\right) \subset V$ of Corollary 5.6.

We require one more property of these sets:

(v) For any $x_{0} \in F_{v}$ there exists $r>0$ such that $F_{v} \cap B\left(x_{0}, r\right)=F_{g} \cap B\left(x_{0}, r\right)$.

Indeed, take any point $x_{0} \in F_{v}$ and suppose the claim $(\mathrm{v})$ is false. Then there exists a sequence of points $F_{g} \backslash F_{v} \ni x_{i} \rightarrow x_{0}$. Denote by $I_{x}$ the straight line segment of length $r$ with center at $x$ and parallel to the vector $\nabla v\left(x_{0}\right)=\nabla g\left(x_{0}\right)$. Evidently, for sufficiently small $r>0$ the equality $I_{x} \cap F_{g}=\{x\}$ holds for any $x \in F_{g} \cap B\left(x_{0}, r\right)$. Then, by construction, $I_{x_{i}} \cap F_{v}=\emptyset$ for sufficiently large $i$. Hence for sufficiently large $i$ either $v>y$ on $I_{x_{i}}$ or $v<y$ on $I_{x_{i}}$. For definiteness, suppose $v>y$ on $I_{x_{i}}$ for all $i \in \mathbb{N}$. In the limit we obtain the inequality $v \geq y=v\left(x_{0}\right)$ on $I_{x_{0}}$. However, this last assertion contradicts (iv). This contradiction finishes the proof of $(\mathrm{v})$.

Obviously, (i)-(v) imply that the set $F_{v}=v^{-1}(y)$ is a compact one-dimensional $\mathrm{C}^{1}$-smooth manifold (without boundary). In other words, $v^{-1}(y)$ is a finite disjoint family of $\mathrm{C}^{1}$ cycles $S_{j}, j=1, \ldots, N(y)$.

To prove the last statement of Theorem 5.1, note that, by well known property of Sobolev functions, $\nabla v$ is an absolutely continuous $\mathbb{R}^{2}$-valued function along almost all coordinate lines. Clearly, if $\nabla g\left(x_{0}\right) \neq 0$, then there exists a $\mathrm{C}^{1}$-smooth coordinate transformation of a neighborhood of $x_{0}$ such that the level sets of $g$ in this neighborhood are transformed into lines parallel to one of the coordinate axes. Using the invariance of Sobolev spaces under smooth coordinate transformations (see $\$ 1.1 .7$ in [28]), we obtain the last assertion of Theorem 5.1.

\section{Application to the level sets of $\mathrm{BV}_{2}$ functions}

The main goal of this section is to prove the following result:

Theorem 6.1. Suppose $v \in \mathrm{BV}_{2}\left(\mathbb{R}^{2}\right)$. Then for almost all $y \in \mathbb{R}$ the preimage $v^{-1}(y)$ is a finite disjoint family of Lipschitz cycles $S_{j}, j=1, \ldots, N(y)$. Moreover, the variation of the tangent vector to each $S_{j}$ (i.e., the integral curvature of $S_{j}$ ) is finite.

Corollary 6.2. Suppose $\Omega$ is a bounded domain in $\mathbb{R}^{2}$ with a Lipschitz boundary and $v \in \mathrm{BV}_{2}(\Omega)$. Then for almost all $y \in \mathbb{R}$ the preimage $v^{-1}(y)$ is a finite 
disjoint family of Lipschitz curves $\Gamma_{j}, j=1, \ldots, N(y)$. Each $\Gamma_{j}$ is a cycle in $\Omega$ or it is a simple arc with endpoints on $\partial \Omega$ (in the last case $\Gamma_{j}$ is transverse to $\partial \Omega$ ). Moreover, the variation of the tangent vector to $\Gamma_{j}$ (i.e., the integral curvature of $\Gamma_{j}$ ) is finite.

Curves of this kind are often called curves of finite turn, and they have been systematically studied in [2] and [36].

For the remainder of the section we fix a function $v \in \mathrm{BV}_{2}\left(\mathbb{R}^{2}\right)$. Let $A_{v}, K_{v}$, $\mu(x), \lambda(x)$, and $\nu(x)$ be as defined in Lemma 4.2 .

Lemma 6.3. For almost all $y \in v\left(\mathbb{R}^{2}\right)$ the following assertions are true:

(i) $v^{-1}(y) \cap A_{v}=\emptyset$.

(ii) For all $x \in v^{-1}(y), \lambda(x) \neq 0 \neq \mu(x)$.

(iii) For all $x \in v^{-1}(y) \cap K_{v}$, both vectors $\lambda(x)$ and $\mu(x)$ are not parallel to $\nu(x)$.

(iv) The intersection $v^{-1}(y) \cap K_{v}$ is at most countable.

(v) $\mathcal{H}^{1}\left(v^{-1}(y)\right)<\infty$.

Proof. The lemma is merely a combination of some of the previous results and standard facts. Thus we only provide a brief sketch:

(i) follows from Theorem 3.2.

(ii) follows from Theorem 4.1.

(iii) follows from the classical one-dimensional version of the Sard Theorem applied to the restriction $\left.v\right|_{L_{i}}$ (see assertions (ii) and (iv) of Lemma 4.2).

(iv) follows from (iii).

(v) follows from the coarea formula.

By connectedness (without additional terms) we mean connectedness in the sense of general topology.

Lemma 6.4 (see, for example, Lemma 2.2 in [20]). Let $\Omega \subset \mathbb{R}^{2}$ be a domain that is homeomorphic to the unit disc and let $G \subset \Omega$ be a subdomain of $\Omega$. Then for each connected component $\Omega_{i}$ of the open set $\Omega \backslash \mathrm{Cl} G$, the intersection $\Omega \cap \partial \Omega_{i}$ is connected.

Lemma 6.5 (see, for example, Lemma 3 in [3]). Suppose $K$ is a compact connected set in $\mathbb{R}^{2}$ and $\mathcal{H}^{1}(K)<\infty$. Then $K$ is arcwise connected.

By arc we mean a set which is homeomorphic to an interval of the straight line.

Lemma 6.6. For any $y \in \mathbb{R}$ satisfying (i)-(v) of Lemma 6.3, for any $x \in v^{-1}(y)$, and for all sufficiently small $r>0$, the connected component $K \ni x$ of the set $B(x, r) \cap v^{-1}(y)$ contains an arc $J \ni x$ with endpoints on $\partial B(x, r)$. Moreover, the arc $J$ intersects at least two connected components of the set $B(x, r) \cap v^{-1}(y) \backslash\{x\}$. 
Proof. We may assume without loss of generality that $x=0, v(x)=0$, and the vector $\nu(x)$ (from Lemmas 4.2 and 6.3) is vertical: $\nu(x)=(0,1)$. Let $L$ be the intersection of the open ball $B(0, r)$ with the horizontal axis: $L=\{(t, 0): t \in$ $(-r, r)\}$. Denote by $A, C$ the endpoints of the segment $L: A=(r, 0), C=(-r, 0)$. If $r>0$ is sufficiently small, then by the differentiability properties recorded in Lemmas 4.2 and 6.3 we infer that the function $v$ is strictly monotone on $L$. For definiteness assume that $v(t, 0)>0$ for $t \in(0, r]$ and $v(t, 0)<0$ for $t \in[-r, 0)$. In particular, $v(A)>0>v(C)$. Let $\Omega_{+}=\{(t, s) \in B(0, r): s>0\}$ and $\Omega_{-}=$ $\{(t, s) \in B(0, r): s<0\}$. Denote by $G$ the connected component of the open set $\left\{z \in \Omega_{+}: v(z)>0\right\}$ such that $A \in \partial G$, and by $\Omega_{1}$ the connected component of the open set $\Omega_{+} \backslash \mathrm{Cl} G$ such that $C \in \partial \Omega_{1}$. Put $K_{+}=\mathrm{Cl}\left(\Omega_{+} \cap \partial \Omega_{1}\right)$. Obviously $0 \in K_{+}, v \equiv 0$ on $K_{+}$, and $K_{+} \cap\left(\partial \Omega_{+}\right) \backslash \mathrm{Cl} \Omega_{-} \neq \emptyset$. Let $D_{+} \in K_{+} \cap\left(\partial \Omega_{+}\right) \backslash \mathrm{Cl} \Omega_{-}$. By Lemma 6.4 the set $K_{+}$is compact and connected, and by (v) of Lemma 6.3 also $\mathcal{H}^{1}\left(K_{+}\right)<\infty$. Then by Lemma 6.5 there exists an arc $J_{+} \subset K_{+}$joining 0 to $D_{+}$. Because $L \cap v^{-1}(0)=\{0\}$ we have equality $J_{+} \cap \mathrm{Cl} \Omega_{-}=\{0\}$. Analogously, there exists a point $D_{-} \in\left(\partial \Omega_{-}\right) \backslash \mathrm{Cl} \Omega_{+}$and an arc $J_{-} \subset \mathrm{Cl}\left(\Omega_{-} \cap v^{-1}(0)\right)$ joining 0 to $D_{-}$so that $J_{-} \cap \mathrm{Cl} \Omega_{+}=\{0\}$. Now $J=J_{+} \cup J_{-}$is the required arc.

Lemma 6.7. For any $y \in \mathbb{R}$ satisfying (i)-(v) of Lemma 6.3 and for any connected component $C$ of $v^{-1}(y)$ there exists a cycle $S \subset C$. Moreover, if there is only one cycle $S \subset C$, then $S=C$.

Proof. To prove the first statement we let $J_{1}$ be a maximal open arc (the latter means it is homeomorphic to the interval $(0,1))$ in $C$. Such an arc exists by Lemma 6.6. Furthermore it follows from (v) of Lemma 6.3 that the inequality $\mathcal{H}^{1}\left(J_{1}\right)<\infty$ holds. So the arc $J_{1}$ has endpoints; denote them by $x$ and $y$. If $x=y$, then there is nothing to prove. The same applies for the case $x \in J_{1}$. If $x \neq y$ and $x \notin J_{1}$ we can continue the arc $J_{1}$ through $x$ by virtue of Lemma 6.6. This contradiction establishes the existence of a cycle $S \subset C$.

To prove the second statement, suppose that $z \in C \backslash S$. Take a maximal open $\operatorname{arc} J_{2}$ in $C$ containing $z$. By the above arguments this arc generates a cycle $S_{2} \neq S$ such that $S_{2} \subset C$.

Corollary 6.8. There exists an at most countable set $Z \subset \mathbb{R}$ such that for any $y \in \mathbb{R} \backslash Z$ satisfying (i)-(v) of Lemma 6.3 all connected components $C$ of $v^{-1}(y)$ are cycles.

Proof. Suppose $y \in \mathbb{R}$ satisfies (i)-(v) of Lemma 6.3 and a connected component $C$ of $v^{-1}(y)$ is not a cycle. Then by Lemma 6.7 the set $\mathbb{R}^{2} \backslash C$ has more than two connected components. By results of [26] (see also [29] and [33]) this is possible only for at most countably many values of $y$.

We need the following maximal inequality and its corollary:

Lemma 6.9 (see, for example, Lemma 1 of $\S 4.8$ in [15]). There exists a constant $C_{5}>0$ such that the following estimate holds for all $t>0$ and $v \in \mathrm{BV}_{2}\left(\mathbb{R}^{2}\right)$ :

$$
\operatorname{Cap}_{1}\left(\left\{x \in \mathbb{R}^{2}: \sup _{r>0} f_{B(x, r)}|\nabla v(y)| \mathrm{d} y \geq t\right\}\right) \leq C_{5} \frac{1}{t}\left\|D^{2} v\right\|\left(\mathbb{R}^{2}\right) .
$$


In view of Lemma 4.2 (iii) we deduce:

Corollary 6.10. For all $t>0$ the following estimate holds:

$$
\operatorname{Cap}_{1}\left(\left\{x \in G_{v}:|\nabla v(x)|>t\right\}\right) \leq C_{5} \frac{1}{t}\left\|D^{2} v\right\|\left(\mathbb{R}^{2}\right) .
$$

Lemma 6.11. For any $\varepsilon>0$ there exist a compact set $F_{\varepsilon} \subset v\left(\mathbb{R}^{2}\right)$ and constants $\delta_{1}, \delta_{2}>0$ such that $\mathcal{L}^{1}\left(v\left(\mathbb{R}^{2}\right) \backslash F_{\varepsilon}\right)<\varepsilon$ and for all $y \in F_{\varepsilon}$ the preimage $v^{-1}(y)$ has the properties (i)-(v) in Lemma 6.3 and additionally:

(vi) For all $x \in v^{-1}(y) \cap G_{v}$ the estimates $\delta_{1}>|\nabla v(x)|>\delta_{2}$ hold.

(vii) Each connected component of the set $v^{-1}(y)$ is a cycle.

Proof. In view of Lemma 6.3 we can choose $F_{\varepsilon}$ so that (i)-(v) are satisfied for all $y \in F_{\varepsilon}$. Property (vi) follows from Theorem 3.1, Lemma 5.4 and Corollaries 4.8 and 6.10. Finally, we obtain property (vii) by use of Corollary 6.8.

Proof of Theorem 6.1. Fix an $\varepsilon>0$ and take the set $F_{\varepsilon}$ from Lemma 6.11. From the above results we have that for each $y \in F_{\varepsilon}$,

$$
v^{-1}(y)=\bigcup_{j=1}^{N(y)} S_{j}(y)
$$

where $S_{j}(y)$ are cycles and $N(y) \in \mathbb{N} \cup\{+\infty\}$.

Take a sequence of functions $v_{i} \in \mathrm{C}^{\infty}\left(\mathbb{R}^{2}\right) \cap \mathrm{W}^{2,1}\left(\mathbb{R}^{2}\right)$ that converges strictly to $v$ in $\mathrm{BV}_{2}\left(\mathbb{R}^{2}\right)$. In particular, we can assume

$$
\begin{gathered}
\nabla v_{i}(x) \rightarrow \nabla v(x) \quad \text { pointwise for all } x \in G_{v}, \\
\left\|D^{2} v_{i}\right\|\left(\mathbb{R}^{2}\right)=\int_{\mathbb{R}^{2}}\left|D^{2} v_{i}(x)\right| \mathrm{d} x \leq 2\left\|D^{2} v\right\|\left(\mathbb{R}^{2}\right) .
\end{gathered}
$$

By the coarea formula,

$\int_{v^{-1}\left(F_{\varepsilon}\right)}|\nabla v(x)| \cdot\left|D^{2} v_{i}(x)\right| \mathrm{d} x=\int_{F_{\varepsilon}} \sum_{j=1}^{N(y)} \int_{S_{j}(y)}\left|D^{2} v_{i}(x)\right| \mathrm{d} \mathcal{H}^{1} \mathrm{~d} y \leq 2 \delta_{1}\left\|D^{2} v\right\|\left(\mathbb{R}^{2}\right)$,

where the last estimate follows from condition (vi) of Lemma 6.11. Consequently there exists a constant $C_{7}$ such that

$$
\int_{F_{\varepsilon}} \sum_{j=1}^{N(y)} \operatorname{Var}\left(\nabla v_{i}, S_{j}(y)\right) \mathrm{d} y \leq C_{7}
$$

where $\operatorname{Var}\left(\nabla v_{i}, S_{j}(y)\right)$ is the variation of $\nabla v_{i}$ on $S_{j}(y)$.

From (6.1) and Lemma 6.11 (viz. the properties (i) and (iv) of Lemma 6.3) it is easy to deduce that

$$
\operatorname{Var}\left(\nabla v, S_{j}(y)\right) \leq \liminf _{i \rightarrow \infty} \operatorname{Var}\left(\nabla v_{i}, S_{j}(y)\right),
$$


and consequently,

$$
\sum_{j=1}^{N(y)} \operatorname{Var}\left(\nabla v, S_{j}(y)\right) \leq \liminf _{i \rightarrow \infty} \sum_{j=1}^{N(y)} \operatorname{Var}\left(\nabla v_{i}, S_{j}(y)\right)
$$

for $y \in F_{\varepsilon}$. Then, by Fatou's lemma,

$$
\int_{F_{\varepsilon}} \sum_{j=1}^{N(y)} \operatorname{Var}\left(\nabla v, S_{j}(y)\right) \mathrm{d} y \leq \liminf _{i \rightarrow \infty} \int_{F_{\varepsilon}} \sum_{j=1}^{N(y)} \operatorname{Var}\left(\nabla v_{i}, S_{j}(y)\right) \mathrm{d} y \leq C_{7} .
$$

Let $\tau$ denote the tangent vector to $S_{j}(y)$. By straightforward geometric considerations and the bounds in Lemma 6.11 (vi) we have

$$
2 \pi \leq \operatorname{Var}\left(\tau, S_{j}(y)\right) \leq \frac{\delta_{1}}{\left(\delta_{2}\right)^{2}} \operatorname{Var}\left(\nabla v, S_{j}(y)\right)
$$

for $1 \leq j \leq N(y)$ and $y \in F_{\varepsilon}$. From the last two formulas we deduce that

$$
N(y)<\infty \text { and } \sum_{j=1}^{N(y)} \operatorname{Var}\left(\tau, S_{j}(y)\right)<\infty
$$

for $\mathcal{L}^{1}$ almost all $y \in F_{\varepsilon}$.

\section{References}

[1] Alberti, G., Csörnyei, M. and Kirchheim, B.: Private communication, July 2010.

[2] Alexandrov, A. D. And Reshetnyak, Yu. G.: General theory of irregular curves. Mathematics and its Applications (Soviet Series) 29, Kluwer Academic Publishers Group, Dordrecht, 1989.

[3] Ambrosio, L., Caselles, V., Masnou, S. and Morel, J.-M.: Connected components of sets of finite perimeter and applications to image processing. J. Eur. Math. Soc. 3 (2001), 39-92.

[4] Bates, S. M.: Toward a precise smoothness hypothesis in Sard's theorem. Proc. Amer. Math. Soc. 117 (1993), no. 1, 279-283.

[5] Bojarski, B., HajŁasz, P. And Strzelecki, P.: Sard's theorem for mappings in Hölder and Sobolev spaces. Manuscripta Math. 118 (2005), 383-397.

[6] Bourgain, J.: A Hardy inequality in Sobolev spaces. Preprint VUB, 1983.

[7] Bourgain, J., Korobkov, M. V. and Kristensen, J.: On the Morse-Sard property and level sets of $\mathrm{W}^{n, 1}$ Sobolev functions on $\mathbb{R}^{n}$. arXiv: 1201-1416 [math.AP], January 6, 2012.

[8] Brown, A. B.: Functional dependence. Trans. Amer. Math. Soc. 38 (1935), 379-394.

[9] Bucur, D., Giacomini, A. And Trebeschi, P.: Whitney property in twodimensional Sobolev spaces. Proc. Amer. Math. Soc. 136 (2008), no. 7, 2535-2545. 
[10] Choquet, G.: L'isométrie des ensembles dans ses rapports avec la théorie du contact et la théorie de la mesure. Mathematica, Timişoara 20 (1944), 29-64.

[11] CsÖRnyei, M., KALiš, J. And ZAJíčEK, L.: Whitney arcs and 1-critical arcs. Fund. Math. 199 (2008), no. 2, 119-130.

[12] De Pascale, L.: The Morse-Sard theorem in Sobolev spaces. Indiana Univ. Math. J. 50 (2001), 1371-1386.

[13] Dorronsoro, J. R.: Differentiability properties of functions with bounded variation. Indiana Univ. Math. J. 38 (1989), no. 4, 1027-1045.

[14] DubovitskiĬ, A. YA.: On the structure of level sets for differentiable mappings of an $n$-dimensional cube into a $k$-dimensional cube (Russian). Izv. Akad. Nauk SSSR Ser. Mat. 21 (1957), no. 3, 371-408.

[15] Evans, L. C. And Gariepy, R. F.: Measure theory and fine properties of functions. Studies in Advanced Mathematics, CRC Press, Boca Raton, FL, 1992.

[16] Federer, H.: Geometric measure theory. Die Grundlehren der mathematischen Wissenschaften 153, Springer-Verlag, New York, 1969.

[17] Figalli, A.: A simple proof of the Morse-Sard theorem in Sobolev spaces. Proc. Amer. Math. Soc. 136 (2008), 3675-3681.

[18] Kolář, J. and Kristensen, J.: Gradient ranges of bumps on the plane. Proc. Amer. Math. Soc. 133 (2005), no. 6, 1699-1706.

[19] Korobkov, M. V.: An analogue of Sard's theorem for $C^{1}$-smooth functions of two variables. Siberian Math. J. 47 (2006), no. 5, 889-895.

[20] Konobkov, M. V.: Properties of the $C^{1}$-smooth functions with a nowhere dense gradient range. Siberian Math. J. 48 (2007), no. 6, 1019-1028.

[21] Korobkov, M. V.: Properties of $C^{1}$-smooth mappings with a one-dimensional gradient range. Siberian Math. J. 50 (2009), no. 5, 874-886.

[22] Korobkov, M. V.: Properties of $C^{1}$-smooth functions whose gradient range has topological dimension 1. Dokl. Math. 81 (2010), no. 1, 11-13.

[23] Korobkov, M. V.: Bernoulli's law under minimal smoothness assumptions. Dokl. Math. 83 (2011), no. 1, 107-110.

[24] Korobkov, M. V., Pileckas, K. and Russo, R.: On the flux problem in the theory of steady Navier-Stokes equations with nonhomogeneous boundary conditions. Arch. Ration. Mech. Anal. (online first), DOI:10/1007/s00205-012-0563-y.

[25] Korobkov, M. V., Pileckas, K. And Russo, R.: The existence theorem for steady Navier-Stokes equations in the axially symmetric case. arXiv:1110.6301v2, [math-ph], 11 Apr 2012.

[26] Kronrod, A.S.: On functions of two variables (Russian). Uspehi Matem. Nauk (N.S.) 5 (1950), 24-134.

[27] LANDis, E. M.: On functions representable as the difference of two convex functions (Russian). Doklady Akad. Nauk SSSR (N.S.) 80 (1951), 9-11.

[28] Maz'ya, V.P.: Sobolev spaces. Springer Series in Soviet Mathematics, SpringerVerlag, Berlin, 1985.

[29] Moore, R. L.: Concerning triods in the plane and the junction points of plane continua. Proc. Nat. Acad. Sci. USA 14 (1928), no. 1, 85-88.

[30] Morse, A. P.: The behavior of a function on its critical set. Ann. of Math. 40 (1939), 62-70. 
[31] Norton, A.: A critical set with nonnull image has large Hausdorff dimension. Trans. Amer. Math. Soc. 296 (1986), 367-376.

[32] Pavlica, D. And ZajíčEK, L.: Morse-Sard theorem for d.c. functions and mappings on $\mathbb{R}^{2}$. Indiana Univ. Math. J. 55 (2006), no. 3, 1195-1207.

[33] Pittman, C. R.: An elementary proof of the triod theorem. Proc. Amer. Math. Soc. 25 (1970), no. 4, 919.

[34] Pogorelov, A. V.: Extrinsic geometry of convex surfaces. Translations of Mathematical Monographs 35, American Mathematical Society, Providence, RI, 1973.

[35] Ponce, A. C. And Van Schaftingen, J.: The continuity of functions with $N$-th derivative measure. Houston J. Math. 33 (2007), no. 3, 927-939.

[36] Reshetnyak, Yu. G.: The theory of curves in differential geometry from the point of view of the theory of functions of a real variable. Russian Math. Surveys 60 (2005), no. $6,1165-1181$.

[37] SARD, A.: The measure of the critical values of differentiable maps. Bull. Amer. Math. Soc. 48 (1942), 883-890.

[38] Whitney, H.: A function not constant on a connected set of critical points. Duke Math. J. 1 (1935), 514-517.

[39] Ziemer, W. P.: Weakly differentiable functions. Graduate Texts in Mathematics 120, Springer-Verlag, New York, 1989.

Received December 15, 2010; revised August 3, 2011.

Jean Bourgain: School of Mathematics, Institute for Advanced Study, Einstein Drive, Princeton, NJ-08540, USA.

E-mail: bourgain@ias.edu

Miknail V. Korobkov: Sobolev Institute of Mathematics, Acad. Koptyug pr., 4, and Novosibirsk State University, Pirogova Str. 2, 630090 Novosibirsk, Russia.

E-mail: korob@math.nsc.ru

Jan Kristensen: Mathematical Institute, University of Oxford, 24-29 St. Giles', Oxford OX1 4AU, United Kingdom.

E-mail: kristens@maths.ox.ac.uk

Korobkov was supported by the Russian Foundation for Basic Research (project no. 08-0100531-a) and by Federal Target Grant "Scientific and educational personnel of innovation Russia" for 2009-2013 (government contract No. P2224). Kristensen was supported by the EPSRC Science and Innovation award to the Oxford Centre for Nonlinear PDE (EP/E035027/1). 